\section{Altérations de l'épissage et maladies rares}

Pierre de la Grange

> La majorité des gènes codant des protéines chez l'homme sont soumis à l'épissage alternatif, le principal mécanisme permettant d'augmenter la diversité du transcriptome et du protéome. La régulation de l'épissage dépend de complexes ribonucléoprotéiques qui composent le splicéosome, ainsi que de protéines régulatrices qui reconnaissent des séquences au niveau des ARN pré-messagers. De plus en plus de mutations au niveau de ces séquences, mais également au niveau de composants du splicéosome ou de facteurs d'épissage, sont décrites pour être responsables du développement de maladies rares. Depuis ces dernières années, des approches de thérapie ciblée sont développées pour restaurer au moins partiellement des événements d’épissage altérés dans le cadre de ces maladies. <

\section{Épissage et épissage alternatif}

Avant les années 2000, le dogme central de la biologie moléculaire était «un gène est à l'origine de la synthèse d'une protéine » et le nombre de gènes codants (des protéines) était estimé à plus de 100000 . Le développement des technologies de séquençage associé à l'essor de la bioinformatique a permis d'affiner l'estimation du nombre de gènes codants chez l'homme. Des études récentes [1] fondées sur l'analyse de données de séquençage massif d'ARN de plusieurs milliers d'échantillons (différents tissus, tumeurs et lignées cellulaires) ont mis en évidence plus de 91000 gènes, dont environ 21000 codants. Ce nombre est proche de celui d'autres espèces comme la souris, le poisson-zèbre ou le nématode, dont le génome présente également plus de 20000 gènes codants. La complexité d'un organisme n'est donc pas uniquement liée au nombre de gènes codants au sein de son génome. Il existe en effet d'autres mécanismes biologiques permettant d'augmenter la diversité du transcriptome et du protéome. Le principal mécanisme permettant d'augmenter cette diversité est

$(\rightarrow)$ Voir également l'épissage alternatif $[41](\rightarrow)$. la Synthèse de G. Dujardin et al., page 1103 de ce numéro
La Figure 1 présente les grandes étapes de la régulation de l'expression des gènes. Un gène peut être à l'origine de la synthèse de plusieurs transcrits distincts traduits en protéines différentes, qui peuvent avoir des fonctions ou des localisations cellulaires différentes. Certains transcrits ont également un rôle régulateur et sont dégradés par la voie du NMD (nonsense-mediated mRNA decay) [2]. Ces transcrits sont reconnus par le NMD parce qu'ils présentent un codon stop prématuré (ou PTC pour premature stop codon).

L'épissage alternatif n'est pas une exception mais plutôt une règle puisque ce mécanisme concerne la très large majorité des gènes humains codants. Si on considère les gènes avec plusieurs exons, $90 \%$ des gènes humains codants sont soumis à épissage alternatif $[3,4]$. II y a en moyenne environ quatre transcrits différents par gène $[3,4]$ et pour un tiers des gènes, l'épissage alternatif est à l'origine de la synthèse d'au moins cinq ARN messagers différents [3, 4]. L'estimation de cette diversité des ARN messagers se fonde sur les connaissances actuelles, et notamment sur les bases de données de transcrits qui restent limitées: en fonction des types cellulaires et tissulaires, des stades de développement, des pathologies et des traitements appliqués (par exemple, siARN [small interfering RNA], composés chimiques, rayonnements ultra-violet, stress cytotoxique, etc.), à peine plus de la moitié seulement des transcrits seraient mis en évidence à ce jour [5]. En considérant la part de transcrits non encore identifiés, les variations génétiques et l'édition (un autre mécanisme permettant l'augmentation de la diversité des transcrits) [6] ainsi que les modifications post-traductionnelles, on pourrait estimer entre 100000 et 1000000 le nombre de protéines différentes chez I'homme. Les conséquences de l'épissage alternatif vont de modifications subtiles à de profonds changements cellulaires. L'épissage peut jouer un rôle: (1) de modification des propriétés cellulaires; (2) de modulation de la transcription; (3) sur la localisation cellulaire des 


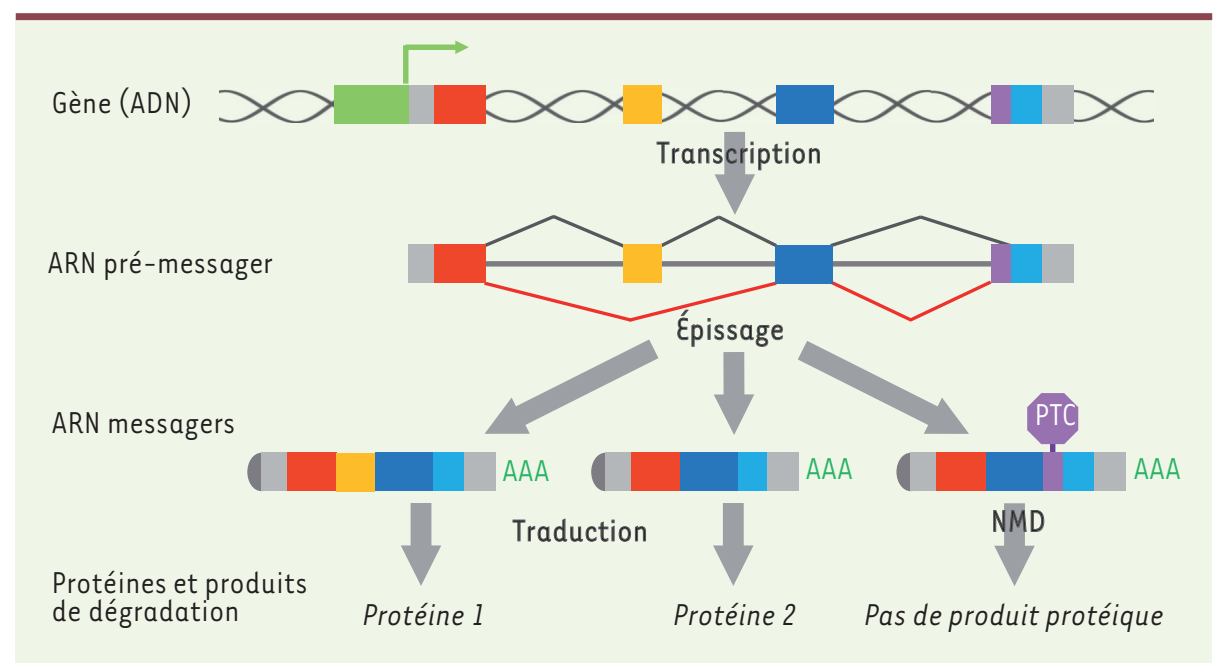

Figure 1. Les grandes étapes de la régulation de l'expression des gènes. La région promotrice du gène est représentée par le rectangle vert; le site d'initiation de la transcription par la flèche verte ; les exons codants en couleur (rouge, jaune, bleu foncé, violet et bleu clair) et les régions non-traduites (UTR pour untranslated region) en gris; l'octogone violet contenant «PTC» (pour premature termination codons) indique la position d'un codon STOP prématuré. La transcription permet la synthèse des molécules d'ARN pré-messagers qui sont ensuite maturés en ARN messa-

gers. Il y a un ajout de la coiffe en 5' et une queue polyA en 3' qui permettent la stabilité des ARN dans le cytoplasme. L'épissage alternatif permet d'obtenir des transcrits avec un contenu en exons différent. Dans cet exemple, l'exon jaune n'est pas inclus dans le deuxième transcrit et il y a un site alternatif d'épissage qui est utilisé pour le dernier exon dans le troisième transcrit. L'insertion de cette région violette introduit un codon STOP prématuré et la dégradation du transcrit par la voie du NMD (non-sens mediated mRNA decay).

protéines; (4) sur leurs propriétés enzymatiques; (5) sur leurs interactions avec d'autres protéines; (6) sur les canaux qu'elles forment ; (7) sur leur stabilité ; ou (8) indirectement sur la régulation de l'épissage en modifiant la composition en exons des transcrits des facteurs d'épissage [7]. De plus en plus d'études montrent également l'importance de la régulation de l'épissage des ARN non codants, notamment dans le cancer [8].

\section{Mécanismes de régulation de l'épissage}

Les exons codants des gènes ne représentent qu'environ $1,5 \% \mathrm{du}$ génome humain. Afin d'être reconnus précisément pour ne pas décaler le cadre de lecture de la traduction, le mécanisme moléculaire de l'épissage nécessite une extrême précision reposant sur le splicéosome, une machinerie composée de plus de 200 protéines distinctes. Le splicéosome est constitué de cinq complexes ribonucléoprotéiques (U1, U2, U4/U6 et U5) qui vont reconnaître plusieurs signaux au niveau de la séquence des ARN pré-messagers (Figure 2A): les sites d'épissage 3 ' et 5' appelés aussi respectivement site accepteur et site donneur; le point de branchement; et la séquence riche en polypyrimidines. La réaction d'épissage se déroule en plusieurs étapes présentées sur la Figure $2 B$. II est maintenant clairement établi que la réaction d'épissage a lieu simultanément à la transcription et qu'un certain nombre de protéines interviennent à la fois dans la régulation de l'épissage et celle de la transcription [9].

Cette reconnaissance des exons par le splicéosome est assortie d'un niveau supplémentaire de régulation qui a pour fonction de renforcer la reconnaissance des sites d'épissage, ou au contraire de la diminuer. En effet, des variants génétiques apparus au cours de l'évolution entraînent l'affaiblissement de sites d'épissage constitutifs ou le renforcement de sites d'épissage cryptiques (normalement peu utilisés). Ils permettent ainsi à la machinerie d'épissage de changer le contenu en exons des ARN messagers synthétisés à partir d'un même gène. Les exons alternatifs ont des sites d'épissage plus faibles (moins bien reconnus par la machinerie d'épissage) que les exons constitutifs (qui sont retrouvés dans la majorité des variants d'épissage). Les séquences d'ARN pré-messager présentent de nombreuses séquences régulatrices, au niveau des exons mais aussi des introns, sur lesquelles les protéines régulatrices de l'épissage (appelées aussi facteurs d'épissage) vont se fixer pour masquer un site ou au contraire renforcer sa reconnaissance (Figure 2C). Les deux grandes familles de facteurs d'épissage sont les protéines riches en acides aminés sérine et arginine, nommées protéines $S R$, et les protéines des particules ribonucléoprotéiques hétérogènes nucléaires (hnRNP). De manière simplifiée, il est souvent admis que les protéines SR se fixent sur les séquences activatrices (ESE, pour exonic splicing enhancer dans les exons, ou ISE, pour intronic splicing enhancer dans les introns) pour favoriser la reconnaissance des sites d'épissage (Figure 2C). À l'inverse, les hnRNP sont généralement connues pour se fixer sur les séquences inhibitrices (ESS, pour exonic splicing silencer dans les exons, ou ISS, pour intronic splicing silencer dans les introns) pour altérer la reconnaissance d'un site en le masquant (Figure 2C). La reconnaissance d'un exon et son inclusion dépend de la combinaison des facteurs d'épissage impliqués (présence et concentration cellulaire). II est par ailleurs maintenant bien établi que d'autres conditions peuvent influencer le choix d'inclusion ou d'exclusion d'exons alternatifs comme des modifications des histones [9], ou la vitesse de transcription [10]. 


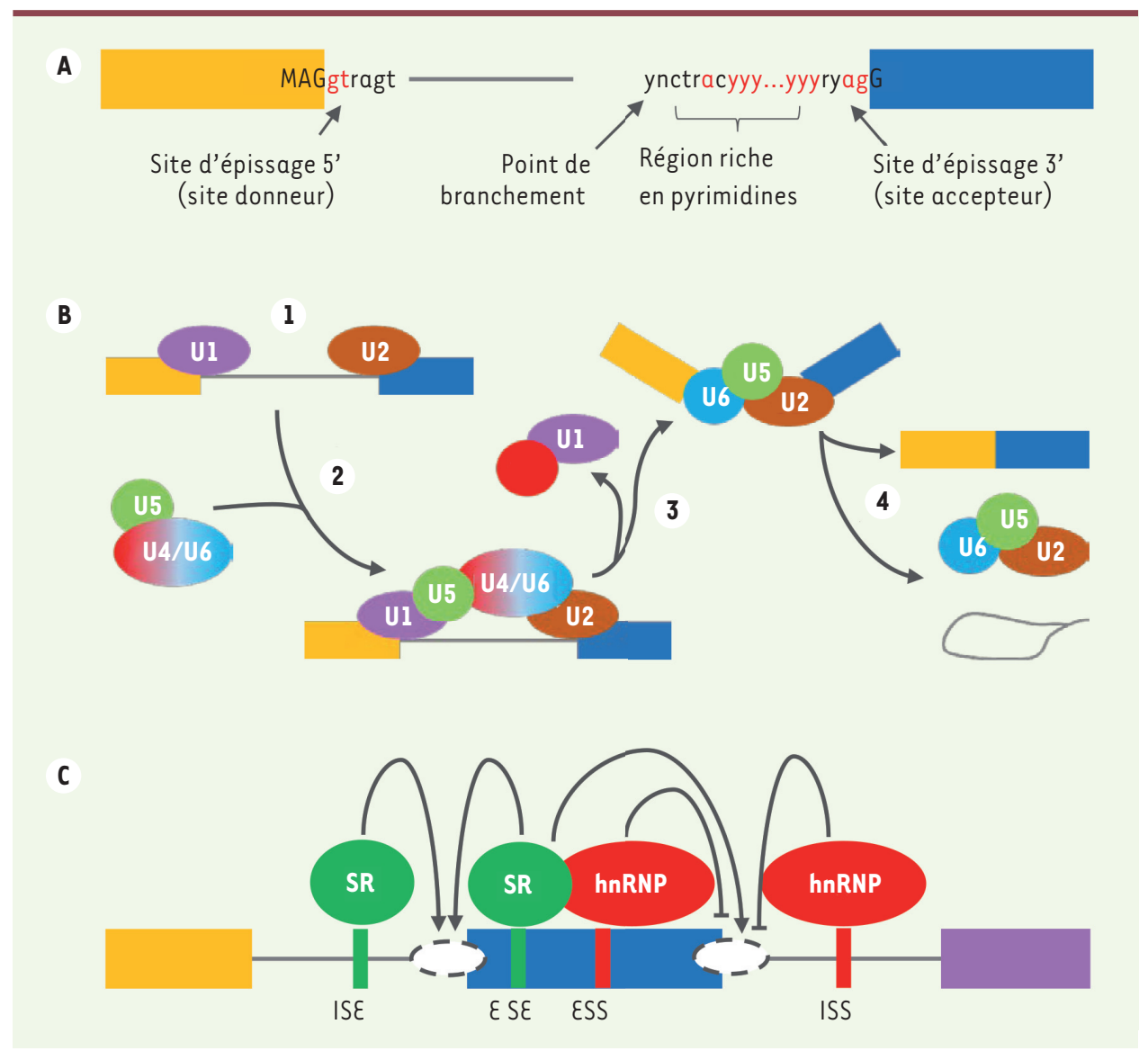

Figure 2. L'épissage. A. Signaux de l'épissage reconnus par les complexes ribonucloprotéiques du splicéosome. Le code IUPAC (International Union of Pure and Applied Chemistry) est utilisé pour décrire la séquence nucléotidiques ( $M: A$ ou $C ; N: A, C$, $G$ ou $T ; R$ : A ou $G ; y: C$ ou $T$ ). $B$. La réaction d'épissage. (1) Le complexe Ul s'associe au site donneur et U2 se fixe sur le point de branchement. (2) Les complexes U4/U5/U6 interviennent: le complexe U6 se fixe sur le complexe U2 tandis que le complexe U5 va se fixer sur l'extrémité 3' de l'exon (proche du site donneur d'épissage) à proximité du complexe U1. (3) Les complexes UI et U4 sont libérés, le complexe U5 glisse sur l'intron et le complexe U6 se fixe sur le site donneur qui est coupé. (4) L'extrémité 3' de l'intron est coupée, ce qui libère l'intron (forme d'un «lasso ») et les exons sont liés. Enfin, le com-

plexe se dissocie. C. La modulation de la reconnaissance des sites d'épissage par les protéines régulatrices de l'épissage. II est souvent admis que les protéines SR (serine/arginine-rich) se fixent sur les séquences activatrices ESE (exonic splicing enhancer) ou ISE (intronic splicing enhancer). À l'inverse, les hnRNP (heterogeneous nuclear ribonucleoprotein) sont généralement connues pour se fixer sur les séquences inhibitrices $\varepsilon S S$ (exonic splicing silencer) ou ISS (intronic splicing silencer).

\section{Dérégulation de l'épissage dans les maladies rares}

En France, une maladie est dite rare lorsqu'elle affecte moins d'un individu sur 2000 . II existe entre 7000 et 8000 maladies rares et, en moyenne, cinq nouvelles sont décrites chaque semaine dans la littérature scientifique. Pour plus de $80 \%$ de ces maladies, une origine génétique a été confirmée. L'importance des variants génétiques dans le contexte des analyses de séquences de patients atteints de maladie d'origine génétique a longtemps été limitée aux régions codantes des transcrits. Seuls les variants prédits pour entraîner un changement d'acide aminé étaient considérés comme des mutations potentielles. Les variants synonymes (des mutations ne modifiant pas l'acide aminé) étaient ainsi écartés, au même titre que les variants localisés en dehors des régions codantes. Il est maintenant établi qu'entre $15 \%$ et $35 \%$ des mutations connues pour être la cause d'une maladie génétique affecteraient l'épissage [11, 12]. Sans a priori sur le fait qu'un variant génétique puisse être ou non une mutation causale d'une maladie génétique, certaines études estiment même à plus de $60 \%$ la proportion de variants qui pourraient affecter l'épissage [13].
Les mutations peuvent affecter l'épissage en agissant en cis (sur le même gène) ou en trans (à distance du gène). Les mutations agissant en cis peuvent altérer un site d'épissage accepteur ou donneur constitutif, ou favoriser la reconnaissance d'un site cryptique, modifier la composition nucléotidique d'une région riche en pyrimidines ou d'un point de branchement, ou altérer une séquence régulatrice de l'épissage. Les mutations agissant en trans peuvent modifier l'efficacité des composants du splicéosome en altérant par exemple un domaine fonctionnel ou domaine d'interaction. Les facteurs d'épissage peuvent également être mutés et entraîner ainsi une altération de la reconnaissance de certains exons. Enfin, d'autres mécanismes d'altération de l'épissage ont été décrits comme responsables de maladies rares, comme par exemple la séquestration de protéines régulatrices de l'épissage due à une répétition de triplets. Les exemples de maladies rares liées à des altérations de l'épissage présentées dans le Tableau / sont détaillés ci-après. 


\begin{tabular}{|c|c|c|c|c|}
\hline Maladie & Prévalence & Gènes $^{a}$ & Type d'altération & $\begin{array}{l}\text { Conséquence } \\
\text { sur la protéine }\end{array}$ \\
\hline Bêta+ thalassémie & $1 / 100000$ & $H B B$ & Site 3'/5' d'épissage (cis) & $\begin{array}{l}\text { Insertion, délétion d'acides } \\
\text { aminés ou protéine tronquée }\end{array}$ \\
\hline $\begin{array}{l}\text { Dystrophie musculaire de } \\
\text { Duchenne }\end{array}$ & $1 / 3300$ (garçons) & $D M D$ & Site 3'/5' d'épissage (cis) & Protéine tronquée \\
\hline Mucoviscidose & $\begin{array}{l}\text { Entre } 1 / 10000 \\
\text { et } 1 / 8000\end{array}$ & CFTR & $\begin{array}{l}\text { Site } 3^{\prime} / 5^{\prime} \text { d'épissage et région } \\
\text { riche en pyrimidines (cis) }\end{array}$ & $\begin{array}{l}\text { Insertion, délétion d'acides } \\
\text { aminés ou protéine tronquée }\end{array}$ \\
\hline Ataxie-télangiectasie & $1 / 100000$ & ATM & Site 3'/5' d'épissage (cis) & $\begin{array}{l}\text { Délétion d'acides aminés ou } \\
\text { protéine tronquée }\end{array}$ \\
\hline Glycogénose de type 2 & $1 / 138000$ & GAA & Site 3'/5' d'épissage (cis) & $\begin{array}{l}\text { Délétion d'acides aminés ou } \\
\text { protéine tronquée }\end{array}$ \\
\hline $\begin{array}{l}\text { Dystrophie musculaire des } \\
\text { ceintures de type lB }\end{array}$ & $1 / 123000$ & LMNA & Site 3'/5' d'épissage (cis) & Protéine tronquée \\
\hline $\begin{array}{l}\text { Lipodystrophie partielle } \\
\text { familiale type } 2\end{array}$ & $\begin{array}{l}200 \text { à } 300 \text { cas } \\
\text { publiés }\end{array}$ & LMNA & Site 3'/5' d'épissage (cis) & Protéine tronquée \\
\hline $\begin{array}{l}\text { Syndrome de Hutchinson- } \\
\text { Gilford }\end{array}$ & $1 / 400000$ & LMNA & Site 3'/5' d'épissage (cis) & Délétion de 50 acides aminés \\
\hline Cardiomyopathies dilatées & $\begin{array}{l}\text { Entre } 1 / 6000 \\
\text { et } 1 / 5000\end{array}$ & LMNA & Site 3'/5' d'épissage (cis) & Insertion de 3 acides aminés \\
\hline Syndrome de Beals & Inconnue & FBN2 & Point de branchement (cis) & Protéine tronquée \\
\hline $\begin{array}{l}\text { Maladie de Niemann-Pick de } \\
\text { type } 1\end{array}$ & $\begin{array}{l}\text { Entre } 1 / 500000 \\
\text { et } 1 / 200000\end{array}$ & $N P C l$ & Point de branchement (cis) & Protéine tronquée \\
\hline $\begin{array}{l}\text { Syndrome du PT long } \\
\text { congénital }\end{array}$ & $1 / 2500$ & KCNH2 & Point de branchement (cis) & Protéine tronquée \\
\hline $\begin{array}{l}\text { Porphyrie érythropoiétique } \\
\text { congénitale }\end{array}$ & $<1 / 1000000$ & UROS & Point de branchement (cis) & Protéine tronquée? \\
\hline Hémophilie B & $1 / 30000$ & $F I X$ & $\begin{array}{l}\text { Région riche en } \\
\text { pyrimidines (cis) }\end{array}$ & Protéine tronquée? \\
\hline $\begin{array}{l}\text { Démence fronto-temporale } \\
\text { et parkinsonisme liée au } \\
\text { chromosome } 17\end{array}$ & $\begin{array}{l}\text { Entre } 1 / 100000 \\
\text { et } 1 / 10000\end{array}$ & MAPT & $\begin{array}{l}\text { Séquence régulatrice de } \\
\text { l'épissage (cis) }\end{array}$ & Délétion d'acides aminés \\
\hline Amyotrophie spinale & $1 / 30000$ & SMN1/SMN2 & $\begin{array}{l}\text { Séquence régulatrice de } \\
\text { l'épissage (cis) }\end{array}$ & Protéine tronquée \\
\hline Rétinites pigmentaires & $\begin{array}{l}\text { Entre } 1 / 10000 \\
\text { et } 1 / 2000\end{array}$ & $\begin{array}{l}\text { PRPF31, } \\
\text { PRPF16, } \\
\text { PRPF8, } \\
\text { PRPF6, } \\
\text { PRPF4, } \\
\text { PRPF3 et } \\
\text { SNRNP200 }\end{array}$ & $\begin{array}{l}\text { Composants du splicéosome } \\
\text { (trans) }\end{array}$ & NA \\
\hline $\begin{array}{l}\text { Anémie sidéroblastique } \\
\text { idiopathique acquise }\end{array}$ & $1 / 100000$ & $S F 3 B 1$ & Facteur d'épissage (trans) & NA \\
\hline $\begin{array}{l}\text { Dystrophie myotonique de } \\
\text { type } 1\end{array}$ & $\begin{array}{l}\text { Entre } 1 / 10000 \\
\text { et } 1 / 2000\end{array}$ & MAPT & Facteur d'épissage (trans) & NA \\
\hline $\begin{array}{l}\text { Dystrophie myotonique de } \\
\text { type } 2\end{array}$ & $1 / 100000$ & ZNF9 & $\begin{array}{l}\text { Séquestration de facteur } \\
\text { d'épissage }\end{array}$ & NA \\
\hline $\begin{array}{l}\text { Sclérose latérale } \\
\text { amyotrophique }\end{array}$ & $\begin{array}{l}\text { Entre } 1 / 100000 \\
\text { et } 1 / 10000\end{array}$ & $\begin{array}{l}\text { C9ORF72, } \\
\text { SOD1, } \\
\text { TARDBP et } \\
\text { FUS }\end{array}$ & $\begin{array}{l}\text { Facteur d'épissage (trans) et } \\
\text { séquestration de facteur d'épissage }\end{array}$ & NA \\
\hline
\end{tabular}

a: la dénomination des gènes est présentée dans le texte. NA : non attribué.

Tableau I. Exemples de maladies rares liées à des défauts d'épissage. 


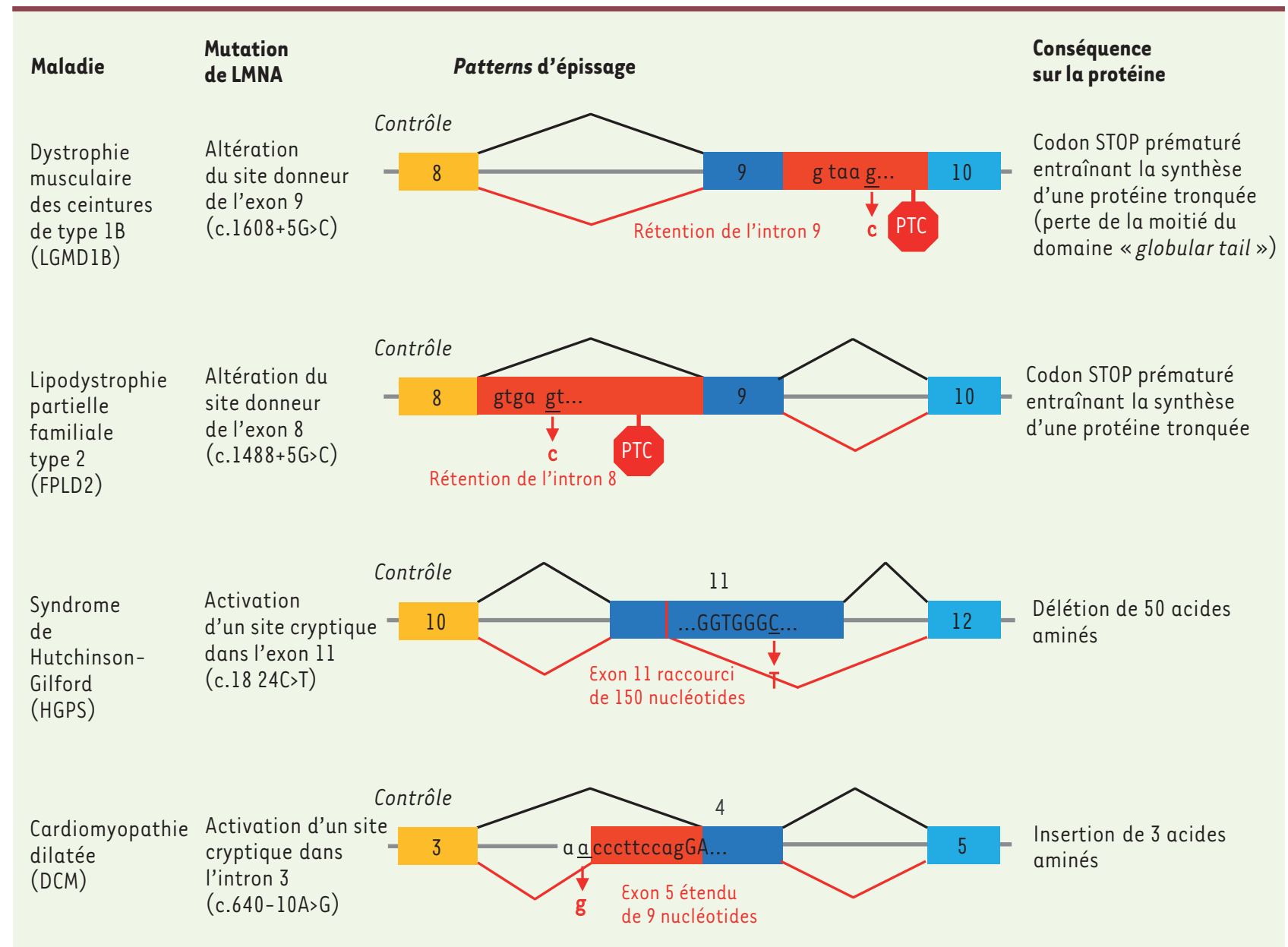

Figure 3. Des altérations de l'épissage d'un même gène peuvent être la cause d'une même maladie : Exemple du gène LMNA. Au milieu de la figure («patterns d'épissage ») sont indiqués en rouge les mutations et les événements d'épissage aberrants qui en résultent. Les octogones rouges contenant «PTC » (pour premature termination codons) indiquent la position de codons STOP prématurés. LMNA : gène de la lamine A/C.

\section{Exemples de mutations agissant en cis au niveau d'un site d'épissage}

Une mutation au niveau d'un site accepteur du gène de la chaîne bêta de l'hémoglobine entraîne la bêta + thalassémie qui est caractérisée par un déficit partiel de synthèse des chaînes de bêta-globine de l'hémoglobine et une anémie ${ }^{1}$ [14]. La dystrophie musculaire de Duchenne est une maladie neuromusculaire caractérisée par une atrophie et une faiblesse musculaire progressive due à une dégénérescence des muscles. La maladie est liée à des mutations affectant l'épissage du gène de la dystrophine [15]. La mucoviscidose est la plus fréquente des maladies génétiques touchant les enfants dans les populations occidentales. Elle est associée à plus de 1250 mutations dans le gène CFTR (cystic fibrosis transmembrane conductance regulator $)^{2}$, dont certaines affectent directement des sites d'épissage [16]. L'ataxie-télangiectasie est une maladie responsable de troubles nerveux et immunologiques. Cette

\footnotetext{
${ }^{1}$ La bêta-thalassémie est caractérisée par un déficit total (Bêta0) ou partiel (Bêta+) de synthèse des chaînes de bêta-globine de l'hémoglobine.

${ }^{2}$ CFTR est un canal ionique permettant le transport des anions chlorure à travers les membranes plasmiques des cellules.
}

maladie est due à l'inactivation du gène ATM (ataxiatelangiectasia mutated) liée à une mutation intronique affectant un site cryptique d'épissage. Un autre exemple de mutation au niveau d'un site d'épissage entraînant une maladie est le cas de la glycogénose de type II qui se traduit notamment par une atteinte des muscles à laquelle s'associe une cardiomyopathie dans la forme infantile. L'analyse génétique du gène GAA (acid alpha-glucosidase) a révélé que la mutation non-sens p.R600 $C^{3}$ affectait l'inclusion de l'exon correspondant [17]. D'autres études ont mis en évidence des variants introniques affectant également l'épissage de ce gène [18]. Contrairement aux exemples présentés précédemment où un gène muté était associé à une seule maladie, un même gène peut être associé à plusieurs maladies selon les mutations qu'il porte. C'est le cas du gène de la lamine $A / C$ (LMNA). Les laminopathies regroupent

\footnotetext{
${ }^{3}$ Substitution d'une arginine $(R)$ en cystéine $(C)$ en position 600 de la protéine.
} 


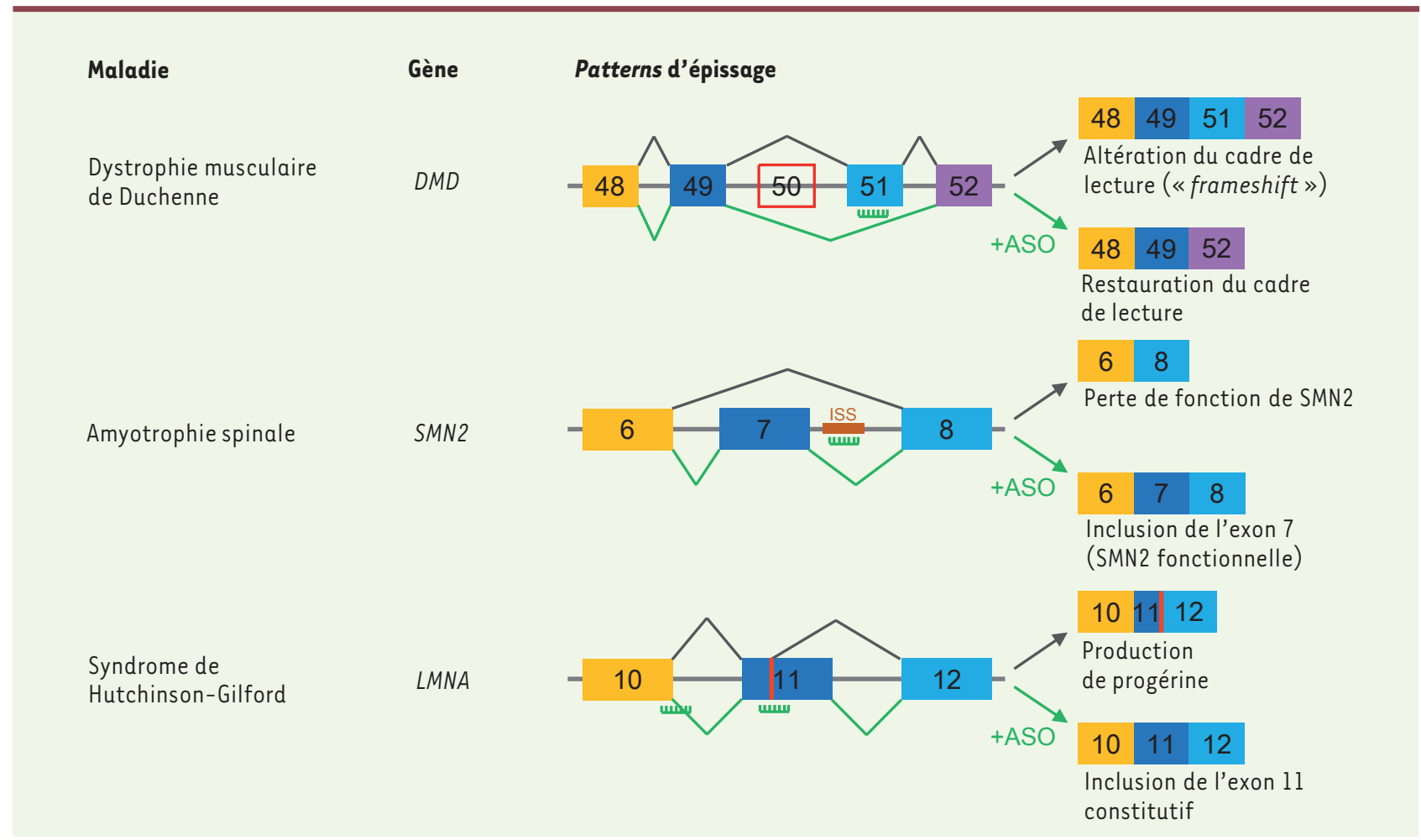

Figure 4. Utilisation des oligonucléotides antisens (ASO) pour corriger les événements d'épissage aberrants. Sur la partie droite de la figure («patterns d'épissage ») sont indiqués en vert les événements d'épissage résultant de l'utilisation des ASO. ISS : intronic splicing silencer; DMD : dystrophie musculaire de Duchenne; SMN2 : survival motor neuron 2 ; LMNA : gène de la lamine A/C.

14 maladies dont des cardiomyopathies, des neuropathies périphériques héréditaires, des lipodystrophies, des dystrophies musculaires et des syndromes de vieillissement prématuré [19]. Des mutations dans des sites donneurs d'épissage du gène LMNA peuvent entraîner des maladies distinctes (Figure 3). Ainsi, la dystrophie musculaire des ceintures de type $1 B$ est caractérisée par une faiblesse musculaire progressive alors que la lipodystrophie partielle familiale type 2 (appelée aussi syndrome de Dunnigan) se caractérise par une absence de tissu adipeux souscutané dans la partie inférieure du corps et une accumulation du tissu adipeux au niveau du visage et du cou [20]. Le syndrome de HutchinsonGilford (ou progéria) est une affection caractérisée par un vieillissement prématuré lié à l'utilisation d'un site 5' d'épissage cryptique au niveau de l'exon 11 de la LMNA [21]. D'autres mutations au niveau du site 3' de l'exon 4 entraînent des cardiomyopathies dilatées [22].

\section{Exemples de mutations agissant en cis} au niveau d'un point de branchement

Les mutations décrites au niveau du point de branchement sont rares. À ce jour, seule une douzaine de mutations sont référencées. Le syndrome de Beals (appelé aussi arachnodactylie congénitale avec contractures) est une maladie du tissu conjonctif dont les symptômes sont proches de ceux du syndrome de $\operatorname{Marfan}^{4}$. Cette maladie est due

${ }^{4}$ Le syndrome de Marfan est une maladie systémique du tissu conjonctif qui se caractérise par une combinaison variable de manifestations cardiovasculaires, musculo-squelettiques, ophtalmologiques et pulmonaires. à une mutation au niveau du point de branchement de l'intron 30 du gène FBN2 qui code la fibrilline 2. La maladie de Niemann-Pick de type 1 est une maladie lysosomale due au déficit de l'activité de la sphingomyélinase acide. Ce défaut est responsable de la surcharge des cellules en sphingomyéline. Des patients atteints de cette pathologie sont porteurs de la mutation c.882-28A>G du gène NPCl (Niemann-Pick disease, type $(1)$ qui altère le point de branchement de l'intron 6 [23]. Le syndrome du QT long congénital ${ }^{5}$ est une maladie cardiaque héréditaire. Le point de branchement de l'intron 9 du gène KCNH2 (potassium voltagegated channel subfamily $H$ member 2) est affecté par une mutation $-28 A>G$ chez certains patients [24]. Un dernier exemple de mutation altérant un point de branchement est la porphyrie érythropoïétique congénitale (appelée aussi maladie de Günther). Depuis sa description à la fin du XIX ${ }^{e}$ siècle, environ 200 cas ont été rapportés dans la littérature. La maladie se manifeste le plus souvent dès la naissance par une photosensibilité cutanée extrêmement sévère et mutilante. La mutation

\footnotetext{
${ }^{5}$ Le syndrome du QT long congénital se caractérise, sur l'électrocardiogramme de surface, par un allongement de l'intervalle entre les signaux $\rho$ et $T$ (temps de systole ventriculaire) et par un risque élevé d'arythmies potentiellement mortelles.
} 
c.661-31T>G du gène UROS (uroporphyrinogen III synthase) entraîne une altération du point de branchement du dernier intron [25].

Exemples de mutations agissant en cis au niveau d'une région riche en pyrimidines

Il existe également un certain nombre de mutations localisées au niveau de la région riche en pyrimidines. L'exemple le plus connu est la mucoviscidose où des mutations altèrent cette région en 5' de l'exon 9 et entraînent une modulation de la sévérité de la maladie [26]. Dans le cas de l'hémophilie $B$, qui est caractérisée par un déficit en facteur IX (un facteur de coagulation) entraînant des hémorragies spontanées ou prolongées, la mutation c.392-8T>G du gène $F 9$ (codant le facteur $\mathrm{IX}$ ) entraîne l'exclusion de l'exon 5 due à une altération du point de branchement de l'intron 4 [27].

Exemples de mutations agissant en cis au niveau d'une séquence régulatrice de l'épissage

Des mutations introniques, ainsi que des mutations exoniques, nonsynonymes ou synonymes, peuvent affecter les séquences régulatrices de l'épissage (ESE, ESS, ISE et ISS). C'est par exemple le cas dans la démence fronto-temporale avec parkinsonisme liée au chromosome 17 (DFTP-17). Dans cette maladie, des mutations au niveau de l'exon 10 du gène MAPT (microtubule-associated protein tau), qui code la protéine $\mathrm{Tau}^{6}$, peuvent non seulement entraîner une altération de la force du site d'épissage 5' de cet exon, mais aussi affecter des séquences ESE au sein de cet exon. Des séquences ESS du gène MAPT sont également affectées par des mutations chez certains patients [28]. Dans le cas de l'amyotrophie spinale (maladie neuromusculaire caractérisée par une faiblesse musculaire progressive due à la dégénérescence et la perte des motoneurones), une mutation dans l'exon 7 du gène SMN(survival motor neuron) I entraîne l'exclusion de cet exon et une protéine non-fonctionnelle. Chez l'homme, ce gène présente une autre copie appelée $S M N 2$ qui diffère par un variant synonyme $C>T$ dans I'exon 7. Ce variant altère non seulement une séquence $\varepsilon S \varepsilon$ sur laquelle se fixe la protéine SR SF2/ASF (serine/arginine-rich splicing factor 2), mais entraîne également la création d'une séquence $\mathrm{ESS}$ sur laquelle se fixe la protéine hnRNPAl (heterogeneous nuclear ribonucleoprotein Al). Cela induit le saut de l'exon 7 dans certains transcrits : la protéine résultante est non-fonctionnelle et ne permet pas de compenser la perte de fonction de SMN1 [29].

\section{Exemples de mutations agissant en trans}

D'autres mutations associées à des maladies rares altèrent la régulation de l'épissage en agissant en trans. C'est le cas par exemple dans certaines rétinites pigmentaires qui constituent un groupe de troubles oculaires héréditaires entraînant une perte progressive de la vision. II a été montré que les patients atteints de ces maladies portent des mutations au niveau des gènes codants du splicéosome comme PRPF4 (pre-MRNA processing factor 4) [30]. Des facteurs d'épissage peuvent également porter des mutations et entraîner des maladies. C'est le

${ }^{6}$ La protéine Tau stabilise les microtubules au niveau des neurones. cas de mutations dans le gène SF3BI (splicing factor $3 b$ subunit 1) qui sont associées à l'anémie sidéroblastique idiopathique acquise qui appartient au groupe des syndromes myélodysplasiques [31].

D'autres maladies rares sont dues à des altérations entraînant indirectement des défauts d'épissage. C'est le cas de la dystrophie myotonique de type 1 caractérisée par une myotonie et par une atteinte multisystémique. Cette maladie est due à une anomalie d'épissage des exons 2, 6 et 10 du gène MAPT suite à une inactivation des facteurs MBNL (muscleblind like splicing regulator) 1, MBNL2 et CELF2 (CUGBP, Elav-like family member 2) [32]. Dans le cas de la dystrophie myotonique de type 2 qui est également une affection multisystémique (faiblesse musculaire, myotonie, atteinte cardiaque et cataracte), il s'agit d'une expansion de séquences CCTG dans l'intron 1 du gène ZNF9 (zinc finger protein 9) qui entraîne la séquestration de la protéine régulatrice de l'épissage MBNL1. Ce recrutement protéique incorrect conduit à des épissages aberrants de plusieurs transcrits. Environ $10 \%$ des cas de sclérose latérale amyotrophique sont familiaux. Cette maladie neurodégénérative est caractérisée par une paralysie musculaire progressive due à une dégénérescence de certains motoneurones. Des mutations ou des altérations comme des expansions de microsatellites affectant plusieurs gènes impliqués directement ou indirectement dans la régulation de l'épissage (FUS [fused in sarcoma], SODI [superoxide dismutase 1], TDP-43 [TAR-DNA binding protein 43], C9ORF72 [chromosome 9 open reading frame 72], etc.) lui ont été associées [33].

\section{Approches thérapeutiques}

Étant donné le nombre important de maladies liées à des mutations affectant l'épissage, des approches thérapeutiques pour moduler ou corriger ces altérations ont été développées ces dernières années dont certaines sont en cours d'essais cliniques [34]. Les deux principales stratégies utilisées à l'heure actuelle sont l'utilisation de petites molécules et les oligonucléotides antisens.

\section{Les petites molécules}

Par criblage à haut-débit, plusieurs petites molécules ont été identifiées pour augmenter la quantité de protéines SMN (survival motor neuron) dans le cadre de l'amyotrophie spinale [35]. C'est également le cas pour la dystrophie myotonique de type 1 où des composés chimiques pourraient empêcher la séquestration de MBNL (muscleblind like splicing regulator) 1 et restaurer ainsi les défauts d'épissage [36]. 


\section{Les oligonucléotides antisens}

Les oligonucléotides antisens ou «ASO » permettent une modulation ciblée de l'épissage de leur cible. Ces oligonucléotides courts sont complémentaires de leur séquence cible et se fixent sur un site d'épissage ou une séquence régulatrice de l'épissage (Figure 4). Cette approche est développée dans le cadre de la dystrophie musculaire de Duchenne où des ASO ciblent l'ESE de l'exon 51 pour entraîner son exclusion et restaurer ainsi la phase de lecture et la production d'une protéine fonctionnelle [37]. Des approches similaires ont également été utilisées dans le cas de la progéria en ciblant l'exon 10 et l'exon 11 de LMNA [38]. Plus récemment, des ASO ont été développés pour cibler un ISS dans l'intron 7 de SMN2 dans le cadre de l'amyotrophie spinale [35].

\section{Conclusion}

L'épissage est le mécanisme principal qui permet l'augmentation de la diversité de nos cellules. C'est aussi un mécanisme complexe, de par sa régulation et sa plasticité : dans des conditions physiologiques, un même gène peut être à l'origine de la synthèse de plusieurs ARN messagers avec des contenus en exons différents, par exemple selon les tissus, les stades de développement, entre ethnies [39] ou même entre individus [40]. D'un point de vue physiopathologique, les exemples cités dans cette synthèse ne représentent qu'une petite sélection de maladies rares dont la cause est liée à une ou plusieurs mutations affectant l'épissage. Cette synthèse n'a pas non plus abordé l'ensemble des conséquences liées aux dérégulations de l'épissage, notamment les cancers. La compréhension des mécanismes de régulation de l'épissage est donc d'une importance capitale. Cette meilleure compréhension permettra également d'améliorer les thérapies fondées sur la modulation de l'épissage. $\diamond$

\section{SUMMARY}

Deregulation of pre-messenger RNA splicing and rare diseases Most of protein-coding human genes are subjected to alternative pre-mRNA splicing. This mechanism is highly regulated to precisely modulate detection of specific splice sites. This regulation is under control of the spliceosome and several splicing factors are also required to modulate the alternative usage of splice sites. Splicing factors and spliceosome components recognize splicing signals and regulatory sequences of the pre-mRNAs. These splicing sequences make splicing susceptible to polymorphisms and mutations. Examples of associations between human rare diseases and defects in premessenger RNA splicing are accumulating. Although many alterations are caused by mutations in splicing sequence (i.e., cis acting mutations), recent studies described the disruptive impact of mutations within spliceosome components or splicing factors (i.e., trans acting mutations). Following growing of knowledge regarding splicing regulation, several approaches have been developed to compensate for the effect of deleterious mutations and to restore sufficient amounts of functional protein. $\diamond$
LIENS D'INTÉRÊT

Pierre de la Grange est co-fondateur et directeur scientifique de la société GenoSplice technology.

\section{RéFÉRENCES}

1. Lyer MK, Niknafs YS, Malik R, et al. The landscape of long noncoding RNAs in the human transcriptome. Nat Genet $2015 ; 47: 199-208$.

2. Lykke-Andersen $\mathrm{S}$, Jensen $\mathrm{TH}$. Nonsense-mediated mRNA decay : an intricate machinery that shapes transcriptomes. Nat Rev Mol Cell Biol 2015 ; 16 : 66577.

3. De la Grange P, Dutertre M, Correa M, Auboeuf D. A new advance in alternative splicing databases: from catalogue to detailed analysis of regulation of expression and function of human alternative splicing variants. BMC Bioinformatics $2007 ; 8: 180$.

4. Yates A, Akanni W, Amode MR, et al. Ensembl 2016. Nucleic Acids Res 2016 ; 44 : D710-6.

5. De la Grange P, Gratadou L, Delord M, et al. Splicing factor and exon profiling across human tissues. Nucleic Acids Res 2010 ; 38 : 2825-38.

6. Licht K, Jantsch MF. Rapid and dynamic transcriptome regulation by RNA editing and RNA modifications. J Cell Biol 2016 ; 213 : 15-22.

7. Kelemen 0 , Convertini $P$, Zhang Z, et al. Function of alternative splicing. Gene 2013 ; 514 : 1-30.

8. Redis RS, Vela LE, Lu W, et al. Allele-specific reprogramming of cancer metabolism by the long non-coding RNA CCAT2. Mol Cell 2016 ; 61 : 520-34.

9. Naftelberg S, Schor IE, Ast G, Kornblihtt AR. Regulation of alternative splicing through coupling with transcription and chromatin structure. Annu Rev Biochem 2015; 84 : 165-98.

10. Moehle $\varepsilon A$, Braberg H, Krogan NJ, Guthrie C. Adventures in time and space : splicing efficiency and RNA polymerase II elongation rate. RNA Biol 2014 ; $11: 313-9$.

11. Mort M, Sterne-Weiler T, Li B, et al. MutPred splice : machine learning-based prediction of exonic variants that disrupt splicing. Genome Biol 2014 ; 15 : R19.

12. Lim KH, Ferraris L, Filloux $M E$, et al. Using positional distribution to identify splicing elements and predict pre-mRNA processing defects in human genes. Proc Natl Acad Sci USA $2011 ; 108$ : 11093-8.

13. López-Bigas N, Audit B, Ouzounis C, et al. Are splicing mutations the most frequent cause of hereditary disease? FEBS Lett $2005 ; 579: 1900-3$.

14. Busslinger M, Moschonas N, Flavell RA. $\beta$ + thalassemia : aberrant splicing results from a single point mutation in an intron. Cell $1981 ; 27: 289-98$.

15. Fletcher S, Meloni PL, Johnsen RD, et al. Antisense suppression of donor splice site mutations in the dystrophin gene transcript. Mol Genet Genom Med $2013 ; 1: 162-73$.

16. Ramalho AS, Beck S, Penque D, et al. Transcript analysis of the cystic fibrosis splicing mutation 1525-1G>A shows use of multiple alternative splicing sites and suggests a putative role of exonic splicing enhancers. J Med Genet $2003 ; 40$ : e88.

17. Maimaiti M, Takahashi S, Okajima K, et al. Silent exonic mutation in the acid-alpha-glycosidase gene that causes glycogen storage disease type II by affecting mRNA splicing. J Hum Genet 2009 ; $54: 493-6$.

18. Zampieri S, Buratti $\varepsilon$, Dominissini $S$, et al. Splicing mutations in glycogenstorage disease type II : evaluation of the full spectrum of mutations and their relation to patients' phenotypes. Eur J Hum Genet 2011 ; 19 : 422-31.

19. Luo YB, Mastaglia FL, Wilton SD. Normal and aberrant splicing of LMNA. J Med Genet $2014 ; 51: 215-23$.

20. Morel CF, Thomas MA, Cao H, et al. A LMNA splicing mutation in two sisters with severe Dunnigan-type familial partial lipodystrophy type 2. J Clin Endocrinol Metab 2006; 91 : 2689-95.

21. Eriksson M, Brown WT, Gordon LB, et al. Recurrent de novo point mutations in lamin A cause Hutchinson-Gilford progeria syndrome. Nature 2003 ; 423 : 293-8.

22. Otomo J, Kure S, Shiba T, et al. Electrophysiological and histopathological characteristics of progressive atrioventricular block accompanied by familial dilated cardiomyopathy caused by a novel mutation of lamin A/C gene. J Cardiovasc Electrophysiol 2005 ; 16 : 137-45.

23. Di Leo $E$, Panico F, Tarugi $P$, et al. A point mutation in the lariat branch point of intron 6 of NPCl as the cause of abnormal pre-mRNA splicing in NiemannPick type C disease. Hum Mutat $2004 ; 24: 440$.

24. Crotti L, Lewandowska MA, Schwartz PJ, et al. A KCNH2 branch point mutation causing aberrant splicing contributes to an explanation of genotype-negative long QT syndrome. Heart Rhythm 2009 ; 6 : 212-8. 


\section{RÉFÉRENCES}

25. Bishop DF, Schneider-Yin X, Clavero S, et al. Congenital erythropoietic porphyria : a novel uroporphyrinogen III synthase branchpoint mutation reveals underlying wild-type alternatively spliced transcripts. Blood $2010 ; 115: 1062-9$.

26. Tsui LC, Dorfman R. The cystic fibrosis gene : a molecular genetic perspective. Cold Spring Harb Perspect Med $2013 ; 3$ : a 009472

27. Montejo JM, Magallon M, Tizzano $\varepsilon$, Solera J. Identification of twenty-one new mutations in the factor IX gene by SSCP analysis. Hum Mutat $1999 ; 13: 160-5$.

28. Niblock M, Gallo JM. Tau alternative splicing in familial and sporadic tauopathies. Biochem Soc Trans $2012 ; 40: 677-80$.

29. Cartegni L, Hastings ML, Calarco JA, et al. Determinants of exon 7 splicing in the spinal muscular atrophy genes, SMN1 and SMN2. Am J Hum Genet $2006 ; 78: 63-77$.

30. Chen $X$, Liu Y, Sheng $X$, et al. PRPF4 mutations cause autosomal dominant retinitis pigmentosa. Hum Mol Genet 2014 ; 23 : 2926-39.

31. Papaemmanuil $\varepsilon$, Cazzola M, Boultwood J, et al. Somatic SF3B1 mutation in myelodysplasia with ring sideroblasts. N Engl J Med 2011 ; 365 : 1384-95.

32. Carpentier C, Ghanem D, Fernandez-Gomez FJ, et al. Tau exon 2 responsive elements deregulated in myotonic dystrophy type I are proximal to exon 2 and synergistically regulated by MBNLI and MBNL2. Biochim Biophys Acta 2014 ; 1842 : 654-64.

33. Ling SC, Polymenidou M, Cleveland DW. Converging mechanisms in ALS and FTD : disrupted RNA and protein homeostasis. Neuron $2013 ; 79: 416-38$.

34. Kole R, Krainer AR, Altman S. RNA therapeutics : beyond RNA interference and antisense oligonucleotides. Nat Rev Drug Discov 2012 ; 11 : 125-40.

35. Faravelli I, Nizzardo M, Comi GP, Corti S. Spinal muscular atrophy : recent therapeutic advances for an old challenge. Nat Rev Neurol $2015 ; 11: 351-9$.
36. Childs-Disney JL, Stepniak-Konieczna $\varepsilon$, Tran T, et al. Induction and reversal of myotonic dystrophy type 1 pre-mRNA splicing defects by small molecules. Nat Commun $2013 ; 4$ : 2044

37. Kole R, Krieg AM. Exon skipping therapy for Duchenne muscular dystrophy. Adv Drug Deliv Rev 2015 ; 87 : 104-7.

38. Osorio FG, Navarro CL, Cadiñanos J, et al. Splicing-directed therapy in a new mouse model of human accelerated aging. Sci Transl Med 2011 ; 3 : 106 ral07.

39. Li JW, Lai KP, Ching AK, Chan TF. Transcriptome sequencing of Chinese and Caucasian population identifies ethnic-associated differential transcript abundance of heterogeneous nuclear ribonucleoprotein K (hnRNPK). Genomics 2014 ; 103 : 56-64.

40. Stein S, Lu ZX, Bahrami-Samani $\varepsilon$, et al. Discover hidden splicing variations by mapping personal transcriptomes to personal genomes. Nucleic Acids Res $2015 ; 43: 10612-22$

41. Dujardin G, Daguenet $\varepsilon$, Bernard DG, et al. L'épissage des ARN prémessagers : quand le splicéosome perd pied. Med Sci (Paris) 2016 ; $32: 1103-10$

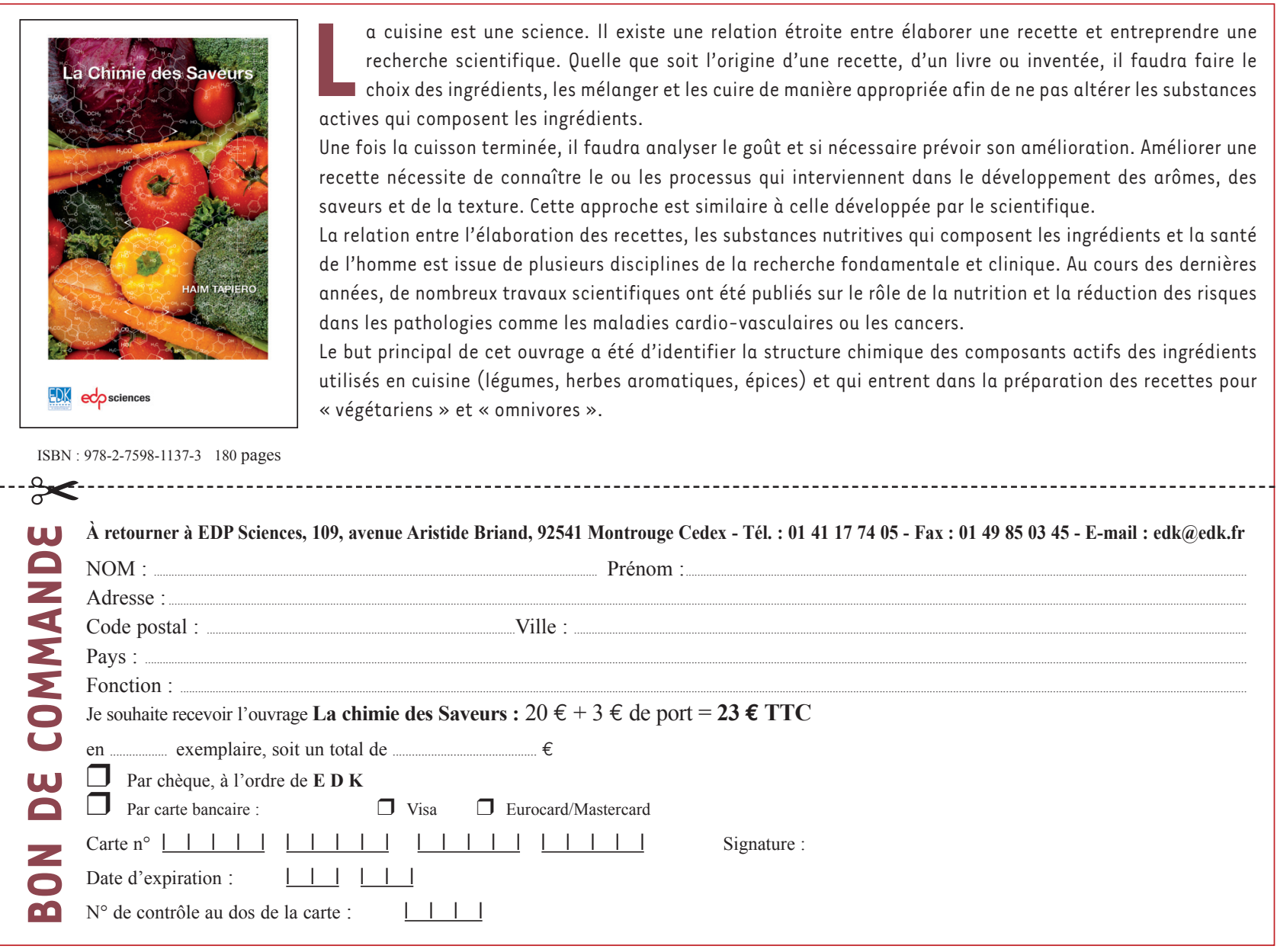

TIRÉS À PART

P. de la Grange 